\title{
Comunicar la humanización de la atención hospitalaria. Un ejercicio de responsabilidad social en los hospitales de Madrid $^{1}$
}

\section{Communicating the humanisation of hospital care. An exercise in social responsibility in Madrid's hospitals}

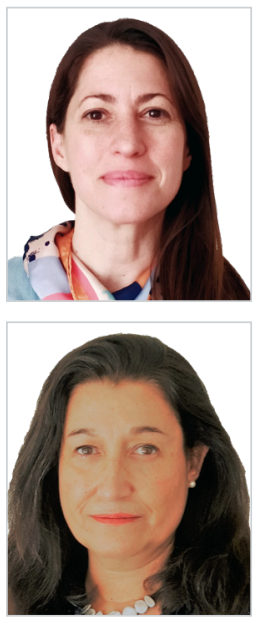

Graciela Lamouret Colom. Licenciada en Ciencia Política por la Universidad de Buenos Aires, Máster en Comunicación Política e Institucional del Instituto Universitario Ortega y Gasset y Máster en Comunicación de las Organizaciones por la Universidad Complutense de Madrid. Durante más de 15 años ha desempeñado su labor en diferentes áreas de comunicación del sector público de Argentina: Oficina de Discursos de Presidencia de la Nación, coordinadora de Comunicación Institucional de la Secretaría de la Gestión Pública, jefa de Prensa del Jefe de Gabinete de Ministros de la Nación y asesora de Prensa en el Senado de la Nación.

Universidad Complutense de Madrid, España

gralamou@ucm.es

ORCID: 0000-0002-1052-3118

María Teresa García Nieto. Profesora Titular de Universidad, doctora y licenciada en Ciencias de la Información y licenciada por la Facultad de Ciencias Políticas y Sociología, ha sido Vicedecana de la Facultad de Ciencias de la Información de la Universidad Complutense de Madrid y Directora del título de Comunicación en el Centro de Estudios Superiores Felipe II de la misma universidad. Es coordinadora de diversos proyectos de investigación, de innovación educativa y consultoría en comunicación; y miembro de varios comités científicos editoriales y de los Comités de Evaluación de Títulos Universitarios de las Agencias para la Calidad del Sistema Universitario en varias comunidades autónomas.

Universidad Complutense de Madrid, España

xyz@ccinf.ucm.es

ORCID: 0000-0002-6235-511X

Recibido: 13/01/2020 - Aceptado: 28/04/2020

Received: 13/01/2020 - Accepted: 28/04/2020

\section{Resumen:}

La humanización constituye, sin duda alguna, un compromiso socialmente responsable de los hospitales, un deber ineludible para lograr unas relaciones públicas excelentes con los pacientes y sus familiares. Nuestro propósito es conocer cómo se comunica la humanización de la atención sanitaria en cuatro hospitales públicos de la Comunidad de Madrid, que sirven de muestra cualitativa, y

\section{Abstract:}

Humanization is, undoubtedly, a socially responsible commitment for hospitals, an inescapable duty to achieve excellent public relations with patients and their families. Our goal is to learn how the humanization of health care is communicated in four public hospitals in the Community of Madrid, which serve as a qualitative sample, and to explore the perception that healthcare

1 Artículo desarrollado bajo el Proyecto "Universidad y sociedad: comunicación e integración en empresas e instituciones públicas y organizaciones no lucrativas”. Universidad Complutense de Madrid. 2016-2020.

Cómo citar este artículo:

Lamouret Colom., G.; García Nieto, M. T. (2020). Comunicar la humanización de la atención hospitalaria. Un ejercicio de responsabilidad social en los hospitales de Madrid. Doxa Comunicación, 30, pp. 187-210.

https://doi.org/10.31921/doxacom.n30a10 
explorar la percepción que tienen sobre ella los profesionales sanitarios. Para ello, tomamos como punto de partida el Plan de Humanización de la atención sanitaria presentado por la Consejería de Sanidad en 2016. Los resultados del análisis documental, las entrevistas en profundidad a los responsables de humanización de estos hospitales, y los grupos de discusión con médicos y personal de enfermería, revelan que el proceso de humanización se presenta dispar en dichos hospitales madrileños, que la comunicación constituye una herramienta fundamental para alcanzar sus objetivos, y la necesidad de optimizar las acciones comunicativas para conseguir los mejores resultados.

\section{Palabras clave:}

Humanización; comunicación hospitalaria; responsabilidad social sociosanitaria; relaciones públicas excelentes; atención sanitaria; atención centrada en el paciente. professionals have of it. To this end, we take as our starting point the Plan for the Humanization of Health Care presented by the Consejería de Salud [Regional Ministry of Health] in 2016. The results of the documentary analysis, in-depth interviews with those responsible for humanization in four hospitals and discussion groups with doctors and nurses show that the humanization process is uneven in these hospitals in Madrid, that communication is a fundamental tool to achieve its objectives, and the need to optimize.

\section{Keywords:}

Humanisation; hospital communication; social and health responsibility; excellent public relations; healthcare; patientcentred care.

\section{Introducción}

El Instituto Nacional de la Salud de España (INSALUD), en octubre de 1984, puso en marcha el primer Plan de la Humanización de la Asistencia Hospitalaria. Este plan, que comenzó implantándose en 15 hospitales, se asentaba en un hecho constatado: "la enfermedad genera, tanto en el paciente como en su entorno familiar y social, una situación de indefensión que le hace sentirse desvalido, por lo que necesita de un sistema sanitario lo más humano posible" (Ministerio de Sanidad y Consumo, 1984: 19). Desde entonces, los hospitales españoles han ido adoptando diversas medidas para optimizar la atención sanitaria, con resultados desiguales.

Treinta y dos años más tarde, en 2016, la Consejería de Sanidad de la Comunidad de Madrid establecía el Plan de Humanización de la Asistencia Sanitaria, ante una situación no muy alentadora: "tristemente existe deshumanización y despersonalización a la hora de prestar la asistencia y los cuidados a las personas" (Subdirección General de Humanización de la Asistencia Sanitaria, 2016: 25).

Este plan, de cuatro años, plantea la humanización de la atención sanitaria como la forma más idónea de relacionar el hospital con los pacientes y sus familiares. Y destaca, además, la importancia de la comunicación como estrategia más eficaz para el perfeccionamiento de esa relación.

La humanización y su comunicación afectan a todo el sistema de salud. Sin embargo, los hospitales constituyen las organizaciones más complejas de la ordenación sanitaria, tanto por sus estructuras, como por sus relaciones. Y los pacientes y sus familiares componen un público de esencial interés para el hospital, porque interactúan de manera directa con numerosos profesionales: médicos, personal de enfermería, técnicos de laboratorio, auxiliares, personal de atención al público, administrativos, camareros, personal de limpieza, entre otros. Por ese motivo, el hospital va representar el foco de nuestro trabajo. 
Los pacientes y sus familiares, sin embargo, no configuran el único público del hospital. Los hospitales, especialmente los públicos, son organizaciones que conciernen a toda la ciudadanía, aunque muchas personas jamás hayan necesitado ser atendidas en un centro hospitalario. Además, los hospitales públicos dependen de la Consejería de Sanidad, a la que rinden cuentas y con cuyos gestores necesariamente se relacionan. Así, aunque las autoridades de los hospitales de la Comunidad de Madrid tienen autonomía para ciertas acciones y decisiones, la estructura jerárquica autonómica es la que marca las líneas generales de acción y entre ellas se encuentra el Plan de Humanización de la Atención Sanitaria.

En este trabajo proponemos estudiar los elementos y procesos de comunicación que, en el marco del citado Plan de Humanización, se están implementando en varios hospitales de la Comunidad de Madrid que forman parte de la red de hospitales públicos de esta Comunidad y, por tanto, del sector sanitario público español.

La sanidad pública española destaca por su prestigio, tal como indican los rankings internacionales sobre la calidad sanitaria, que la ubican en los primeros puestos del mundo (Lancet, 2018). Además, el sector sanitario público cuenta con una alta aprobación por la población española. Así lo refleja el Barómetro Sanitario del Centro de Investigaciones

Sociológicas (CIS) de 2018, con los siguientes datos referidos a la población de todo el territorio nacional:

- El 68,3 por ciento de los españoles opina que el sistema sanitario público funciona bien o bastante bien: el 47,1 por ciento respondió "bien, pero son necesarios algunos cambios"; y el 21,2 por ciento respondió "bastante bien".

- Preguntados sobre dónde acudirían en caso de requerir asistencia sanitaria (para el entrevistado o para un familiar), pudiendo elegir, el 68,2 por ciento de los encuestados prefiere la atención pública.

- Y en caso de tener que ser ingresados en un hospital, el 67 por ciento prefiere el sistema público, y sólo el 26,3 por ciento se inclinan por el privado.

El citado Barómetro Sanitario del CIS, al referirse a la Comunidad de Madrid, asevera:

- Un 48,9 por ciento de los madrileños considera que el sistema sanitario funciona bien, aunque son necesarios algunos cambios.

- Un 26,2 por ciento cree fundamentales algunos cambios, aunque reconoce el buen funcionamiento de procedimientos concretos.

- Y un 3,9 por ciento expresa la necesidad de rehacer todo el sistema sanitario público debido a su mal funcionamiento.

Y los habitantes de esta Comunidad señalan los principales problemas de su sistema de salud:

- En primer lugar, las listas de espera para ser atendidos (85,7 por ciento).

- En segundo lugar, la congestión de las urgencias de los hospitales (75,8 por ciento).

- Y un 15,1 por ciento de los madrileños considera que el principal problema es el trato de los profesionales médicos, cuando la media nacional en este aspecto alcanza el 19,7 por ciento (CIS, 2018). 


\section{La comunicación hospitalaria}

Los hospitales, como organizaciones que quieren comunicarse con sus públicos, poseen una serie de características distintivas, derivadas de su función principal de curar y cuidar, respondiendo a una necesidad básica de la persona que en términos generales no se puede eludir o aplazar. Es un servicio cuya prestación, al menos en España, está garantizada por el Estado y es universal (Costa, 2012a: 176).

Por un lado, los hospitales son organizaciones en las cuales la mayor parte de los empleados tiene contacto directo con el paciente y con sus familiares. Por otro, el servicio hospitalario entraña un alto grado de proximidad con el público, implica la presencia constante del paciente durante todo el proceso de la prestación del servicio sanitario. Es decir, la misma prestación del servicio hospitalario sólo puede producirse en el encuentro con el "otro", con el paciente, "una vez que la comunicación y el diálogo se entabla a partir de la interacción” (Quintana, Castillo, Carretón, 2018: 86). Así, el diálogo con los prestadores de ese servicio constituye un rasgo propio y diferencial del ser humano, e implica la capacidad de comunicar, de entender, de querer y de elegir (Quintana, Castillo, Carretón, 2018).

De otra parte, y a pesar de los esfuerzos preventivos, la enfermedad resulta imprevisible, por ello el requerimiento del servicio sanitario se considera una actividad sujeta a incertidumbre. Y ello provoca en ocasiones "picos" en las necesidades de atención sanitaria, difícilmente predecibles, como ocurre ante hechos trágicos o situaciones epidémicas (Costa, 2012b).

En cualquier caso, e independientemente de las circunstancias concretas, los públicos de una organización hospitalaria, como los de cualquier empresa, pueden clasificarse en tres categorías dependiendo de su relación con la entidad: públicos internos, públicos externos y públicos intermedios o ambivalentes. Los públicos internos componen la organización, forman parte de ella y mantienen con ella una relación directa y permanente. Los públicos externos, en cambio, se relacionan de forma esporádica con la organización. Y los públicos intermedios, son aquellos quienes, sin tener una relación permanente y de pertenencia a la organización, sí la afectan o se ven afectados de manera más o menos directa por ella. Además, como señalan Viñarás y Cabezuelo, esta clasificación, derivada de la planificación estratégica de las relaciones con los públicos, es igualmente válida para la identificación de los destinatarios de la RSC de las organizaciones (Viñarás y Cabezuelo, 2012: 58).

Los ciudadanos son público externo del hospital. Sin embargo, si un ciudadano requiere un servicio hospitalario, se convierte en usuario, en paciente, adquiriendo así la condición de público intermedio, como les sucede también a sus cuidadores y familiares. Y es necesario reflexionar sobre la situación por la cual ese ciudadano se convierte en público intermedio. Se encuentra en una situación de fragilidad y estrés, en la cual las habilidades de comunicación interpersonal de los profesionales sanitarios influyen en la motivación del paciente y pueden condicionar sus decisiones, como seguir un tratamiento o acudir a las revisiones establecidas (Medina, 2017: 357). La comunicación centrada en el paciente resulta ser la forma de comunicación interpersonal más eficaz para el tratamiento médico, aunque "apenas ha sido explorada por el ámbito facultativo nacional" (Salcedo, 2012: 42). 
Por otro lado, el público interno del hospital agrega diversos grupos de personas: personal médico, estudiantes en formación, biólogos, técnicos, matronas, enfermeros, psicólogos, auxiliares, personal de administración y servicios y los grupos de investigación, entre otros (Costa, 2012b: 111).

En este contexto, la gestión estratégica de la comunicación en las relaciones entre el hospital y sus públicos internos desempeña un papel fundamental, y en ella "resulta imprescindible la implicación, colaboración, motivación y cohesión de todo el personal de la organización para alcanzar la excelencia en la gestión” (García Nieto, 2012b: 129). Y, en este sentido, la comunicación interna cumple tres funciones en los centros hospitalarios: función identificadora, función integradora y función motivadora (García Nieto, 2012b: 133). Desde la Teoría General de Sistemas (von Bertalanffy, 1976) se puede afirmar que "un hospital es un sistema en permanente interacción con su entorno y en constante adaptación en sus procesos internos" y donde prevalece el necesario esfuerzo de los directivos para "garantizar la integración y cohesión del personal" (García Nieto, 2012b: 130).

\section{Relaciones públicas excelentes y humanización}

Sólo es posible pensar la humanización de la atención sanitaria en el marco epistemológico de la excelencia de las relaciones con los públicos, y siempre enmarcada en la ineludible atención centrada en el paciente. La humanización constituye un principio básico e imprescindible del quehacer hospitalario y representa un valor propio de los preceptos más elementales de la responsabilidad social sanitaria. La humanización es clave en la relación con las personas que se sienten inseguras por cuestiones de salud. Por eso, es preciso despojarla de cualquier connotación filantrópica que la dotaría de un carácter discrecional y arbitrario, voluntario y opcional, algo impensable cuando se trata de las relaciones con los pacientes.

\subsection{La excelencia en las relaciones con los públicos}

Como es sabido, la Teoría de la Excelencia de las Relaciones Públicas surge a partir de los estudios realizados en 1985 a instancias de la Fundación de Investigación de la IABC (International Association of Business Communicators), conducentes a explicar "el valor de las relaciones públicas para una organización y a identificar las características de la función de las relaciones públicas que incrementan su valor” (Grunig y Grunig, 2008: 327).

Una de las aportaciones fundamentales de la Teoría de la Excelencia es la definición de los cuatro modelos de las Relaciones Públicas. Estos modelos, basados en la investigación empírica, y reflejo de las diferentes formas de llevar a la práctica las relaciones con los públicos a lo largo de la historia, desde principios del siglo veinte, se extrapolan en el tiempo presente con los elementos que de cada modelo se pueden observar en el contexto actual. De manera que, como señalan sus autores, los diferentes modelos pueden encontrarse vigentes en las organizaciones, aplicados en distintas proporciones. Sin embargo, añaden, el modelo deseable para el desarrollo de las relaciones públicas es el llamado modelo de relaciones públicas excelentes, de comunicación bidireccional y resultados equilibrados (Grunig, 1992; Grunig y Hunt, 2003).

La excelencia se refiere a un conjunto de características que definen la efectividad de una organización. Y una organización es efectiva cuando "consigue alcanzar las metas elegidas en consulta con sus públicos - metas que sirven a los 
intereses de la organización y de estos destinatarios estratégicos" (Grunig y Grunig, 2008: 328). Son atributos y prácticas que ayudan a "construir relaciones a largo plazo, de calidad, con los destinatarios estratégicos" (Grunig, Grunig y Ehling, 1992: 86).

Este cuarto modelo de relaciones excelentes, el modelo bidireccional simétrico, "brinda una norma teórica de cómo deberían practicarse las relaciones públicas para ser éticas y efectivas, característica de una gestión excelente de la comunicación" (Grunig y Grunig, 1992: 285).

La comunicación en el modelo simétrico bidireccional "consiste más en un diálogo que en un monólogo" (Grunig y Hunt, 2003: 75). Y la singularidad determinante de las relaciones simétricas radica en el equilibrio de resultados entre las dos partes, logrando ajustar la relación entre la organización y su público: “antes que la persuasión, es el entendimiento el principal objetivo de las relaciones públicas” (Grunig y Grunig, 1992: 289).

La práctica de las relaciones públicas excelentes se basa en la investigación y utiliza la comunicación para gestionar el conflicto y mejorar el entendimiento con los públicos estratégicos. Se fundamenta en la negociación y el compromiso, con unos claros presupuestos éticos. "Las relaciones públicas excelentes son aquellas que añaden valor a la organización, ayudan a la organización a alcanzar sus objetivos, colaboran para que la organización establezca sinergias con sus públicos y promueven la construcción de procesos relacionales teniendo en cuenta el interés público" (Grunig, Grunig, Aparecida-Ferrari, 2015: 14).

Resumiendo:

- Las relaciones públicas excelentes son necesariamente relaciones socialmente responsables. "Las relaciones públicas excelentes son una política socialmente responsable que ha de regir el comportamiento de una persona natural o jurídica, con el fin de alcanzar y mantener su mutuo entendimiento con los diferentes públicos" (García Nieto, 2012a: 101). Atender las demandas, las necesidades y los intereses de los públicos estratégicos se convierte en una prioridad de sus objetivos.

- El modelo simétrico bidireccional de relaciones excelentes con los públicos es el modelo idóneo y el más conveniente para el progreso de la humanización de la atención sanitaria. Pues existe una clara confluencia entre el modelo de relaciones excelentes, simétricas y bidireccionales, y la atención sanitaria centrada en el paciente.

- Las relaciones públicas excelentes y la responsabilidad social corporativa (RSC) son inseparables, por ello la humanización de la atención sanitaria sólo es posible desde los planteamientos éticos y socialmente responsables de la organización.

\subsection{La RSC sanitaria. El doble desafío de la RSC en la atención sanitaria}

La atención sanitaria española viene experimentando en las últimas décadas un importante crecimiento de las acciones específicas de responsabilidad social corporativa propias de su ámbito de servicio público, en las cuales la humanización juega un papel esencial. 
Recordemos que la RSC "es arrogada por una organización cuando ésta responde a la necesidad de asumir las consecuencias de su gestión, de sus políticas, de sus procedimientos, de sus conductas y de su comunicación, en relación con los diferentes grupos sociales, contemporáneos o futuros, afectados por ella a corto, medio o largo plazo" (García Nieto, 2012a: 94).

La RSC del sector sanitario, por las características particulares del servicio que en él se presta, posee un componente social y humano más acentuado que en las organizaciones de otros sectores.

Si recordamos los niveles de la responsabilidad en las organizaciones formulados por Preston y Post (1975) y Grunig (Grunig y Hunt, 1984, 2003, Grunig, 1992), podríamos afirmar que la responsabilidad de las organizaciones sanitarias, y en concreto de los hospitales, implica tanto la denominada "responsabilidad pública", como la "responsabilidad social". La atención sanitaria per se representa una garantía del bienestar social y de la mejora de la calidad de vida. Los profesionales de la sanidad, y por ende las organizaciones en las que trabajan, no sólo contraen los compromisos de curar, remediar y cuidar a los pacientes, y asumir sus consecuencias, sino también y sobre todo de hacerlo bien y siempre desde los principios de la vocación de servicio a las personas, de servicio social, del respeto al paciente, y de la ética, propios de la responsabilidad social.

\subsubsection{El componente social de la atención sanitaria y la RSC}

El servicio sanitario que los hospitales prestan a las personas es en sí mismo un ejercicio de RSC y el objetivo principal de la responsabilidad social sanitaria es el paciente, por ello algunos gestores hospitalarios la denominan responsabilidad social centrada en el paciente (Redacción Médica, 2014). El impacto emocional y social de la atención sanitaria, el nivel elevado de especialización de los profesionales sanitarios, y el contacto directo de los empleados con los pacientes son "factores que obligan a los responsables de comunicación de los centros hospitalarios a reflexionar sobre un nuevo concepto de RSC que se adapte al hospital y que respete la esencia de la responsabilidad social organizacional. Este nuevo concepto debe basarse en tres aspectos principales: el valor prioritario de la comunicación interpersonal, el protagonismo del paciente, y el compromiso con el conocimiento científico" (Medina, 2012a: 82). "Satisfacer médica y emocionalmente al paciente es una de las principales acciones de RSC que puede emprender un hospital. Por ello, entre las iniciativas de RSC que lleva a cabo un hospital debe figurar la formación de sus empleados en habilidades de comunicación interpersonal" (Medina, 2012a: 83).

Pero si centramos el foco en la sanidad pública, debemos referirnos necesariamente al marco de la responsabilidad social en el sector público, un marco que ha ido consolidándose en la última década. Al aproximarnos a la RS en el sector público comprobamos que ha sido objeto de comentarios discordantes por parte de algunos autores, para quienes las instituciones públicas "ya eran de interés público". Aunque se reconoce que no basta "con hacer las cosas bien desde un punto de vista normativo y de calidad, sino que, además, la manera como se procede tiene impactos económicos, sociales, ambientales, laborales y reputacionales, que hay que gestionar porque también forman parte del interés público y de la responsabilidad que las instituciones han de asumir ante el conjunto de la sociedad" (Canyelles, 2011: 84). En este sentido, "la Administración pública tiene dos enfoques respecto a la RSC: uno, potenciar las buenas prácticas de forma voluntaria y otro, sugerir que es necesario regular el tema" (Rodríguez Cala, 2017: 63) y "requiere que la organización de 
la propia institución incorpore la RS en sus políticas de gestión y procedimientos, implicando a las partes interesadas y consiguiendo así el compromiso de los directivos" (Rodríguez Cala, 2017: 65).

En esta línea, la responsabilidad social en las organizaciones del sector público puede definirse como "aquellas políticas de carácter voluntario por parte de las instituciones públicas orientadas al bien común y a generar un valor social (con beneficios sociales, económicos y medioambientales convergentes), que exceden los mínimos exigidos por ley, y alineadas con las expectativas y necesidades de sus stakeholders" (Bustos, 2017: 134).

\subsection{Humanización y RSC}

La Humanización y la Responsabilidad Social Corporativa en el sector sanitario van de la mano, se entrecruzan, comparten objetivos y tareas, se confunden en algunos espacios, y crecen juntas, al menos en los últimos años. Como se señala en el texto del Marco estratégico de promoción de la Responsabilidad Social Sociosanitaria de la Comunidad de Madrid, la RSC "está directamente relacionada con la perspectiva ética y de humanización de las organizaciones sanitarias" (Dirección General de Coordinación de la Atención al Ciudadano y Humanización de la Asistencia, 2017: 3).

Por su parte, el Instituto de Innovación y Desarrollo de la Responsabilidad Social Sociosanitaria (Inidress), en su decálogo de la Responsabilidad Social Sociosanitaria, afirma, en su segundo precepto, que "la humanización de la asistencia es un valor trascendental e imprescindible en la Responsabilidad Social Sanitaria” (Inidress, 2017: 7).

Algunos autores opinan que las iniciativas de RSC pueden servir como acicates para la implementación de planes de humanización en la asistencia sanitaria. Así lo expresa el grupo de trabajo sobre humanización sanitaria, formado por investigadores de la Cátedra Inditex de Responsabilidad Social de la Universidad de A Coruña, profesionales de la salud del Centro Terapéutico Xerontolóxico A Veiga, y gestores sanitarios de la Xerencia de Xestión Integrada del Área Sanitaria de Santiago de Compostela (Gil Paz, et al. 2018). Este grupo de trabajo manifiesta la necesidad de "poner en valor los departamentos de responsabilidad social de las áreas sanitarias... en lo relativo a acciones en pos de la humanización" (p.60), y concluyen que "los postulados de la responsabilidad social y su comunicación eficaz podrían constituir una excelente estrategia hacia el logro de la humanización de la atención sanitaria” (p.54). Las acciones de RSC y la humanización de la atención sanitaria son dos procesos que comparten espacios de acción en el hospital. Comparten destinatarios y el común objetivo de progresar hacia las relaciones excelentes con los públicos.

Algunas actuaciones de RSC de los propios hospitales son claramente acciones de humanización de la atención sanitaria. Sirva de modelo, el denominado "Proyecto Plata", un plan de RSC destinado a la implantación de una plataforma de voluntariado para el acompañamiento del paciente al alta. Este proyecto, emprendido por la Red Sanitaria de $\mathrm{RSC}^{2}$, basado en los datos de los servicios de atención al paciente de seis grandes hospitales de nivel 3 (hospitales que cuentan con la mayoría de las especialidades) de tres comunidades autónomas, se propone dar respuesta a un problema detectado en el 5,21\% de los pacientes en condiciones de alta hospitalaria. El de la falta del apoyo social necesario para que el paciente

2 La Red Sanitaria de RSC, nacida en 2015, es una agrupación sin ánimo de lucro formada por profesionales de distintos hospitales públicos, hoy ya cerca de cuarenta en diferentes comunidades autónomas, con el objetivo implantar políticas de responsabilidad social en el sector sanitario, en cuestiones sociales, medioambientales y de sostenibilidad. Una de sus claves consiste en crear proyectos en los que el paciente sea siempre "lo primero". 
pueda trasladarse del hospital a su residencia habitual. Un problema social, no específico del ámbito sanitario, pero que consecuentemente redunda en el bienestar del paciente (Red Sanitaria de RSC y Grupo Innova-Docencia №176, 2018).

En la Comunidad de Madrid, en concreto, la responsabilidad social sanitaria ha cobrado protagonismo a partir del año 2017, con la presentación por parte de la Consejería de Sanidad, del ya mencionado Marco Estratégico de la Promoción de la Responsabilidad Social Sociosanitaria. En su texto queda clara una idea: la responsabilidad social en "el ámbito sociosanitario adquiere un carácter estratégico, al estar directamente relacionado con la perspectiva ética y de humanización de las organizaciones sanitarias (Dirección General de Coordinación de la Atención al Ciudadano y Humanización de la Asistencia, 2017: 3).

Pero la responsabilidad social sanitaria no sólo proviene de las iniciativas hospitalarias. Encontramos otras acciones de RSC emprendidas por empresas de sectores no sanitarios en apoyo de los hospitales, que también colaboran con la humanización de la atención al paciente. Pensemos, como ejemplo, en aquellas iniciativas destinadas a la mejora de los espacios en los hospitales públicos, o aquellas dirigidas a reducir el impacto traumático de la estancia hospitalaria, o de las intervenciones quirúrgicas, especialmente en los pacientes infantiles.

\subsection{Humanización y atención centrada en el paciente}

Julio Zarco, director de la Fundación Humans y ex Director general de Atención al Ciudadano y Humanización de la Asistencia Sanitaria de la Comunidad de Madrid, afirma que humanizar "significa garantizar la dignidad de la persona cuando más vulnerable se encuentra, es también una forma compasiva de tratar al paciente, de escucha activa, terapéutica y empática, y establecer una relación simétrica entre el profesional y el paciente” (Zarco, 2018a).

José Carlos Bermejo, especialista en humanización y director del Centro San Camilo, en Tres Cantos (Madrid), considera que la atención sanitaria humanizada debe respetar al paciente como persona única e irrepetible, y reconocer "el protagonismo de los pacientes y familiares en los procesos de salud" (Bermejo, 2014: 3). Sin embargo, la humanización de la atención sanitaria exige, además, una comunicación eficaz. El enfermo debe ser "ayudado a comprender su situación con una información clara y precisa", debe conocer las opciones disponibles en cuanto a su tratamiento, "de lo contrario, solo desempeñará un papel pasivo de dependencia" (Bermejo, 2014: 3).

No obstante, algunos profesionales sanitarios ponen en entredicho el término humanización y utilizan en su lugar el de "atención centrada en el paciente" (Zarco, 2018b: 208). Esta expresión, empleada en la literatura anglosajona (King y Hope, 2013; Stewart, 1995, 2001; Greene, Tuzzio y Cherkin, 2012), se refiere a una determinada forma de relación entre el personal sanitario y los pacientes, más sensible a sus necesidades, sus preferencias y valores, a la hora de tomar decisiones, prescribir y aplicar cuidados y tratamientos. La atención centrada en el paciente sólo puede concebirse desde una perspectiva biopsicosocial, superando los esquemas exclusivamente biomédicos, y asentada en un vínculo de confianza entre paciente y médico (King y Hope, 2013; Stewart, 1995, 2001; Greene, Tuzzio y Cherkin, 2012).

En su libro Patient-Centered Medicine: Transforming The Clinical Method (Stewart et al, 2003), un grupo de investigadores de las Universidades de Ontario, Western Ontario y Ottawa, Canadá, atribuyen la enunciación del concepto "Medicina centrada en el paciente” al médico psiquiatra húngaro Michael Balint, quien, con un grupo de colegas, después de estu- 
diar la psicología de la relación entre médicos y pacientes, acuñaría el término y destacaría sus diferencias con la medicina centrada en la enfermedad (Balint, 1979). La medicina centrada en el paciente supondrá una transformación en el método clínico (Stevens, 1974; Tait, 1979) y su posterior perfeccionamiento (Byrne y Long, 1984). El grupo dirigido por Stewart entenderá, además, que el nuevo paradigma, esta renovación en la interacción de los médicos con los pacientes, significará un giro fundamental en la relación de poder: "para brindar una atención centrada en el paciente, el médico debe ser capaz de empoderar al paciente, de compartir el poder en la relación” (Stewart et al, 2003: 5).

En 1995, Stewart publicó un artículo en el que se recogían los resultados de las investigaciones sobre la comunicación médico-paciente, realizadas a lo largo de 25 años. Este estudio longitudinal evidenciaba los problemas derivados de la falta de transmisión de información. Por un lado, la relativa a la información transmitida por el paciente al médico, cuando éste trata de elaborar la imprescindible historia clínica. Y, por otro, la información transmitida por el médico al paciente, cuando el médico pretende explicarle su prescripción y el tratamiento.

En líneas generales, los estudios consultados por Stewart señalaban:

- Un 50 por ciento de los problemas psicosociales y psiquiátricos pasan desapercibidos en las consultas.

- Los médicos interrumpen a los pacientes a los 18 segundos, en promedio, cuando estos exponen los síntomas de sus dolencias.

- El 54\% de los males y el $45 \%$ de las preocupaciones de los pacientes no son expresados en las consultas.

- En un 50 \% de los casos los pacientes y los médicos no coinciden en la identificación de qué es lo esencial de la dolencia. Y como consecuencia, los pacientes se sienten insatisfechos (Stewart, 1995: 1429).

Además, se destaca la importancia de discriminar y explorar de manera diferenciada, de una parte, la enfermedad en sí misma, y de otra, el sentir único y particular del paciente ante sus padecimientos. Stewart insiste en la importancia de la atención centrada en el paciente y destaca sus beneficiosos resultados: menos denuncias por mala praxis, mayor satisfacción de los médicos, mayor satisfacción de los pacientes, mayor seguimiento de los tratamientos, reducción de las preocupaciones y mejor estado psicológico de los pacientes (Stewart et al, 2003: 14).

Años más tarde, en 2012, Greene, Tuzzio y Churkin concluirán que los pacientes con una buena relación con sus médicos se someten a menos estudios clínicos y se ven menos inclinados a iniciar acciones legales ante una mala praxis. Y, asimismo, la atención centrada en el paciente beneficia también a los profesionales médicos quienes resultan ser más eficaces en el tratamiento de sus pacientes. Estos autores expondrán, además, la necesidad de expandir el modelo de la "Medicina centrada en el paciente" a otras relaciones en el contexto sanitario, de manera que no quede reducido a la relación médico-paciente, sino que se amplíe en su aplicación a cualquier tipo de relación interpersonal en el ámbito sanitario, ya sea en la sala de atención primaria, en el hospital, o en cualquier escenario del sistema de salud. 


\section{El Plan de Humanización de la Comunidad de Madrid}

La Ley General de Sanidad, del 25 de abril de 1986, estableció en España un sistema nacional de salud que integraba a las diferentes redes públicas sanitarias existentes hasta su promulgación. Esta ley instauraba la universalidad de la asistencia sanitaria para los españoles y residentes, y determinó un sistema descentralizado en las comunidades autónomas.

La Comunidad de Madrid, con una superficie de $8.022 \mathrm{~km} 2$, es la tercera de España en razón al número de habitantes (6.579.711) y es la más densamente poblada. El Informe Anual del Sistema Nacional de Salud del Ministerio de Sanidad, Consumo y Bienestar Social informaba en 2018 que esta Comunidad cuenta con 108 centros hospitalarios, igualmente la tercera de España. Madrid tiene una tasa de 1,2 hospitales por cada 100 mil habitantes, inferior a la tasa media nacional de 1,7 hospitales por 100.000 habitantes. De esos centros, 37 pertenecen al Sistema Nacional de Salud, con 12.660 camas y 2.267 puestos de atención en hospitales de día (Ministerio de Sanidad, Consumo y Bienestar Social, 2018: 32).

Como ya hemos señalado, la Consejería de Sanidad de Madrid presentó en 2016 el Plan de Humanización para "promover la mejora de la humanización de la asistencia sanitaria" (Subdirección General de Humanización de la Asistencia Sanitaria, 2016: 19). Para su elaboración se identificaron los siguientes grupos de interés: los pacientes, las personas cuidadoras, las asociaciones de pacientes, los ciudadanos, los profesionales, los directivos, otras instituciones, la sociedad (p. 20); se establecieron 10 líneas estratégicas, y se dispusieron 27 programas de actuaciones.

Estos son los ejes estratégicos del Plan:

- Cultura de humanización.

- Información personalizada y acompañamiento.

- Humanización de la asistencia en las primeras etapas de la vida, infancia y adolescencia.

- Humanización en la atención de urgencias.

- Humanización en la hospitalización.

- Humanización en unidades de cuidados intensivos.

- Humanización en la atención de la salud mental.

- Humanización y paciente oncológico.

- Humanización ante el final de la vida.

- Escuela Madrileña de Salud.

La presentación de la primera de estas líneas estratégicas, la Cultura de la humanización, entraña ya aspectos específicamente vinculados con las relaciones públicas y la comunicación. En esta línea, el Plan refiere cuestiones que van a repercutir directamente en la satisfacción del paciente, como las conductas de los profesionales y los directivos, sus actitudes, creencias, usos y costumbres, en la forma y modo de prestar la asistencia sanitaria. Su aplicación se propone transversal en todo su desarrollo, con el propósito de poder evaluar y mejorar la cultura de un determinado hospital, servicio o centro de salud. Igualmente, se destaca la importancia del liderazgo para el cambio de la cultura organizacional y se presenta un "Decálogo de Humanización" con las principales pautas de acción en la relación de los trabajadores del sistema sanitario 
con los pacientes. Este decálogo fue reeditado en abril de 2019 y presentado por la Comunidad de Madrid sumando la contraparte de pautas de acción para los pacientes.

El Plan reconoce también que "la información en la atención sanitaria forma parte de la actividad profesional y representa un valor esencial en todo el proceso asistencial que la organización debe garantizar” (p.81), y destaca, además, la importancia del acompañamiento y el apoyo proporcionado al paciente.

En ese sentido, la información "constituye el requisito indispensable para que el ciudadano adquiera mayor capacidad en el ámbito sanitario para la toma de decisiones, compartida con el equipo sanitario" (p.81), mucho más que la mera transmisión de contenidos e indicaciones.

La segunda línea estratégica de Información personalizada y acompañamiento, directamente dirigida a mejorar las relaciones con los públicos, establece los siguientes propósitos:

- Desarrollar contenidos formativos esenciales, institucionales y homogéneos en toda la red asistencial en materias de información y comunicación personalizadas.

- Establecer un programa que desarrolle los distintos niveles de información institucional en cada centro.

- Optimizar la comunicación institucional.

- Perfeccionar procesos que mejoren la asistencia personalizada.

- Facilitar el acompañamiento del paciente por la persona elegida por él.

El Plan se refiere también al servicio de urgencias: "la atención de urgencias constituye un punto de referencia básico y crítico para el Sistema Nacional de Salud” (p. 99). Es crítico porque el flujo de acceso a estos servicios no puede ser controlado por la organización, y es un punto de referencia básico porque en España, aunque haya otros espacios de atención, "las urgencias hospitalarias constituyen el nivel asistencial más habitual" (p. 99). Por ese motivo, al pensar en la comunicación del hospital como un todo, parece necesario detenernos en esta cuestión.

En la Comunidad de Madrid, en 2018, los servicios de urgencia hospitalarios atendieron a 3.378.220 pacientes (Observatorio Resultados Servicio Madrileño de Salud y Dirección General de Humanización, 2018). Es preciso tener en cuenta que para muchas personas el único contacto durante años con un hospital se localiza en el servicio de urgencias. Por ello, el Plan recoge las dimensiones de satisfacción y de insatisfacción percibida por los pacientes y familiares en los servicios de urgencia, basados en las encuestas de satisfacción que realiza anualmente la Comunidad de Madrid.Y, como se puede observar en el Cuadro 1, los aspectos relativos a la comunicación y la atención se revelan prioritarios. 
Cuadro 1: Dimensiones de la satisfacción e insatisfacción de pacientes y familiares

\begin{tabular}{|l|l|}
\hline \multicolumn{1}{|c|}{ Dimensiones de satisfacción } & \multicolumn{1}{c|}{ Dimensiones de insatisfacción } \\
\hline Información brindada & Falta de información durante la espera \\
\hline Amabilidad y empatía & Demora en la atención tras el primer contacto \\
\hline Actitud de escucha activa, o confianza e intimidad en la conversación & \\
\hline Tiempo de espera percibido & \\
\hline
\end{tabular}

Fuente: Elaboración propia a partir del Plan de Humanización de la Asistencia Sanitaria

Concluye el Plan que "la satisfacción depende, básicamente, de la sensación del paciente de estar siendo bien tratado" (p. 100).

En lo referente a la hospitalización, el Plan la define como "una experiencia emocionalmente intensa tanto para el paciente como para sus familiares y acompañantes" (P. 119), quienes se sienten frágiles en un entorno desconocido, percibido probablemente como hostil, y en una situación en la cual la incertidumbre se convierte en un factor determinante, incluso en el proceso de recuperación.

De la encuesta de satisfacción del Servicio Madrileño de Salud se extraen las principales áreas de mejora en la hospitalización:

- Información facilitada a los pacientes y familiares, tanto asistencial como de trámites de carácter administrativo.

- Accesibilidad de personas con discapacidad.

- Comodidad y confortabilidad del paciente en la habitación.

- Comodidad y confortabilidad de los acompañantes.

- Calidad de la comida.

- Manejo del dolor.

Entre ellas, se mencionan expresamente, por su evaluación negativa, la falta de información y las deficiencias en la comodidad de las habitaciones. Y al estudiar las reclamaciones efectuadas por los pacientes y familiares, comprobamos como destacan las relacionadas con el ámbito de la organización de la asistencia, los circuitos de atención y el trato.

Julio Zarco, ex Director general de Atención al Ciudadano y Humanización de la Asistencia Sanitaria de la Comunidad de Madrid, consultado puntualmente sobre el rol de la comunicación en el diseño del Plan de Humanización, afirma: "La comunicación impregna todo el plan estratégico de humanización, pues la comunicación es fundamental como herramienta entre los profesionales y el sistema sanitario y los pacientes y sus familias. Además, en un marco de responsabilidad social corporativa, la comunicación es un ingrediente fundamental para conseguir una ósmosis entre el hospital y el tejido social donde se encuentra" (Zarco, comunicación digital mantenida el 17 de julio de 2019). 


\section{Objetivo y metodología}

Una vez conocido el Plan de Humanización de la Atención Sanitaria de la Comunidad de Madrid, planteamos la investigación con el objetivo general de averiguar las acciones de comunicación realizadas en cuatro hospitales madrileños, relativas a la humanización de la atención sanitaria, y explorar la percepción que de ella tienen los profesionales sanitarios. Como ya adelantamos, definimos como campo de estudio de nuestra investigación los hospitales públicos de la Comunidad de Madrid, por ser ésta una de las primeras comunidades en implementar planes de humanización. El universo de estudio está constituido por los 37 hospitales pertenecientes al Sistema Nacional de Salud.

Tomamos como unidad de investigación el hospital para conocer los procesos de comunicación en el interior de este tipo de organizaciones, en relación con los diferentes públicos, partícipes y destinatarios de las diversas acciones de humanización de la atención sanitaria. El hospital es, además, receptor y ejecutor de las medidas establecidas por la Comunidad de Madrid según el Plan de Humanización 2016-2019.

La muestra final seleccionada la conforman 4 hospitales, que han expresado su voluntad de colaborar en la investigación, integrantes en la Red Sanitaria de Responsabilidad Social, formada por gestores y directivos de centros hospitalarios españoles, comprometidos en la labor de humanizar la atención sanitaria.

A la hora de seleccionar los hospitales utilizamos los criterios del tamaño y de la ubicación, siendo elegidos los siguientes: el Hospital Clínico San Carlos, el Hospital Universitario 12 de Octubre, el Hospital Universitario Infanta Cristina de Parla y el Hospital de Guadarrama. Aunque la muestra no es suficientemente representativa, los resultados de la investigación sí nos permiten aproximarnos a unas conclusiones razonables y adecuadas.

Cuadro 2: Características de los hospitales seleccionados

\begin{tabular}{|c|c|c|c|c|c|c|c|}
\hline Hospital & $\begin{array}{c}\text { Camas } \\
2018\end{array}$ & $\begin{array}{l}\text { Plantilla } \\
2018\end{array}$ & Complejidad & $\begin{array}{l}\text { Población } \\
\text { referencia }\end{array}$ & $\begin{array}{l}\text { Finalidad } \\
\text { Asistencial }\end{array}$ & $\begin{array}{l}\text { Ingresos } \\
2018\end{array}$ & Tamaño \\
\hline H. U. Clínico San Carlos & 861 & 5016 & Alta & 374.369 & General & 31908 & Grande \\
\hline H. U. 12 de Octubre & 1162 & 6559 & Alta & 446.628 & General & 45053 & Grande \\
\hline Infanta Cristina & 188 & 906 & Baja & 169.612 & General & 8902 & Medio \\
\hline Guadarrama & 144 & 307 & Otros & $*$ & $\begin{array}{c}\text { Geriatría y/o larga } \\
\text { estancia** }\end{array}$ & 1114 & Pequeño \\
\hline
\end{tabular}

Fuente: Elaboración propia en base a Catálogo de Hospitales del Ministerio de Sanidad (2018), la página web de la Comunidad de Madrid, el Observatorio de Resultados del Servicio Madrileño de Salud (2018) y los Programas Gestión de los hospitales participantes

*Guadarrama no tiene población de referencia ya que los ingresados vienen derivados de otros centros de salud de la Comunidad de Madrid.

**En el Catálogo de Hospitales del Ministerio de Sanidad, Consumo y Bienestar Social (2018) el Hospital de Guadarrama es clasificado como "Geriatría y/o Larga Estancia”, mientras que en la página web de la Comunidad de Madrid lo menciona como "hospital de media estancia”. En el Observatorio de Resultados del Servicio Madrileño de Salud (2018) este hospital entra en la categoría de "otros", que incluye "hospitales de apoyo a otros centros, los hospitales de media estancia y los hospitales psiquiátricos”. 
Para cumplir nuestro propósito hemos aplicado las siguientes técnicas:

- Análisis de materiales producidos por los hospitales y por la Consejería de Sanidad.

- Entrevistas en profundidad.

- Grupos de discusión.

Hemos analizado los siguientes materiales:

- Informe de Actividades humanización propuestas para 2018 (Consejería de Sanidad).

- Balance de acciones de humanización 2018 de cada hospital.

- Indicadores Atención al paciente del Observatorio de Resultados del Servicio Madrileño de Salud.

- Programa gestión 2019 de cada hospital.

- Páginas web de cada hospital.

- Materiales de difusión elaborados por los departamentos de comunicación de los hospitales.

Hemos realizado cinco entrevistas en profundidad a responsables de diferentes áreas de gestión de los cuatro hospitales en relación con la humanización, la responsabilidad social, la calidad y la comunicación. Una vez realizadas las entrevistas y obtenidos sus resultados, consideramos necesario estudiar, además, la percepción de los trabajadores sanitarios en los hospitales sobre la humanización y su comunicación. Para ello se aplicaron dos grupos de discusión: uno formado por personal de enfermería y otro por personal médico.

\section{Resultados}

\subsection{Análisis documental}

De toda la documentación analizada, interesa destacar los datos reflejados en el siguiente cuadro: 
Cuadro 3: Indicadores de satisfacción en Atención al Paciente del Observatorio de Resultados del Servicio Madrileño de Salud - Año 2018

\begin{tabular}{|c|c|c|c|c|c|}
\hline & 12 de Octubre & $\begin{array}{c}\text { Clínico San } \\
\text { Carlos }\end{array}$ & $\begin{array}{l}\text { Infanta } \\
\text { Cristina }\end{array}$ & Guadarrama & General \\
\hline Satisfacción global & $85,28 \%$ & $86,70 \%$ & $84,58 \%$ & $82,80 \%$ & $89,0 \%$ \\
\hline Información & $87,21 \%$ & 88,26 & $87,45 \%$ & $83,21 \%$ & $90,28 \%$ \\
\hline Humanización en la hospitalización* & $83,57 \%$ & $85,40 \%$ & $86,70 \%$ & $82,30 \%$ & $87,73 \%$ \\
\hline Humanización en urgencias & $74,30 \%$ & $77,98 \%$ & $75,92 \%$ & No corresponde & $81,5 \%$ \\
\hline Profesionales médicos & $91,30 \%$ & $93,12 \%$ & 90,06 & $87,42 \%$ & $93,05 \%$ \\
\hline Trato y amabilidad profesionales médicos & $92,50 \%$ & $91,93 \%$ & $90,62 \%$ & $90,63 \%$ & $93,45 \%$ \\
\hline Profesionales enfermería & $89,44 \%$ & $91,30 \%$ & $90,06 \%$ & $85,00 \%$ & $92,36 \%$ \\
\hline Habitación & $53,42 \%$ & $78,88 \%$ & $94,97 \%$ & $85,00 \%$ & $77,14 \%$ \\
\hline
\end{tabular}

Fuente: Elaboración propia en base al Observatorio de Resultados del Servicio Madrileño de Salud 2018

*Porcentaje de pacientes satisfechos y muy satisfechos con la humanización en hospitalización.

Para la construcción de este índice de la humanización en la hospitalización se han escogido once preguntas de la encuesta de satisfacción que se agregan en siete dimensiones clave relacionadas con la humanización como son: 1) el trato y la amabilidad de los profesionales 2) la información facilitada en diferentes momentos de la asistencia 3) el tiempo y la dedicación de los profesionales 4) el respeto a la intimidad, 5) la participación en las decisiones de tratamiento y cuidados 6) el silencio por la noche, y 7) el tratamiento del dolor.

\subsection{Las entrevistas en profundidad}

A modo de resumen, indicamos las más relevantes cuestiones derivadas de los resultados de las entrevistas en profundidad: En los hospitales de mayor tamaño:

- La comunicación de la humanización no llega a todo el personal del hospital. Y existen notables diferencias entre unos sectores y otros. Unos, más comprometidos con la humanización, y otros, bastante menos.

- La comunicación interpersonal termina siendo el procedimiento más efectivo para convocar a la participación en las acciones de humanización. 
- Dado su mayor tamaño, pese a las numerosas actividades emprendidas y el gran esfuerzo realizado, los mensajes no llegan a los públicos internos.

- Los hospitales emplean distintas herramientas para comunicar la humanización con los pacientes. El 12 de Octubre destaca por una comunicación más directa y general, con materiales de difusión y consultas a los pacientes. Y el Clínico San Carlos se distingue por un trabajo muy importante con las asociaciones de pacientes.

En los hospitales de mediano y menor tamaño:

- En estos hospitales, el concepto humanización llega a los profesionales sanitarios, aunque, por diversas circunstancias, no alcanza a aplicarse en suficiente medida.

- Algunas áreas del servicio del hospital no suelen reconocer su propia participación en las acciones de humanización, aunque las estén implementando tanto en el contacto con los pacientes, como en la provisión de los servicios que les prestan.

- Curiosamente, los profesionales sanitarios consideran excesiva la cantidad de actividades propuestas en sus hospitales relacionadas con el plan de humanización.

Mostramos las características de la comunicación de la humanización en los hospitales estudiados de manera esquemática mediante el siguiente cuadro. 
Cuadro 4: La comunicación de la humanización en los cuatro hospitales

\begin{tabular}{|c|c|c|c|c|c|}
\hline & $\begin{array}{l}\text { Difusión actividades } \\
\text { humanización }\end{array}$ & $\begin{array}{l}\text { Comunicación } \\
\text { humanización }\end{array}$ & $\begin{array}{l}\text { Principales } \\
\text { problemas }\end{array}$ & Acciones destacadas & $\begin{array}{c}\text { Presencia } \\
\text { humanización en } \\
\text { la página web }\end{array}$ \\
\hline $\begin{array}{l}\text { Clínico } \\
\text { San Carlos }\end{array}$ & $\begin{array}{l}\text { Intranet. } \\
\text { Jefes de servicios. } \\
\text { Correo electrónico } \\
\text { institucional. } \\
\text { Llamada telefónica } \\
\text { personal. }\end{array}$ & $\begin{array}{l}\text { Integrantes comisión } \\
\text { impulsan acciones } \\
\text { en sus áreas. } \\
\text { Referentes } \\
\text { humanización en } \\
\text { servicios. }\end{array}$ & $\begin{array}{l}\text { Personal numeroso. } \\
\text { No llega difusión } \\
\text { ni comunicación a } \\
\text { todo el personal. } \\
\text { Falta comunicación } \\
\text { interna. } \\
\text { Cómo generar } \\
\text { cultura } \\
\text { organización. }\end{array}$ & $\begin{array}{l}\text { Identificación } \\
\text { referentes } \\
\text { humanización en los } \\
\text { servicios. } \\
\text { Intercambio con } \\
\text { asociaciones de } \\
\text { pacientes. }\end{array}$ & \\
\hline 12 de Octubre & $\begin{array}{l}\text { Intranet. } \\
\text { Correo electrónico } \\
\text { institucional. } \\
\text { Jefes de servicios. } \\
\text { Llamada telefónica } \\
\text { personal. }\end{array}$ & $\begin{array}{l}\text { Sesiones generales. } \\
\text { Sesiones por } \\
\text { especialidad o } \\
\text { servicio. } \\
\text { Integrantes comisión } \\
\text { impulsan acciones } \\
\text { en sus áreas. } \\
\text { Intranet. }\end{array}$ & $\begin{array}{l}\text { Personal numeroso. } \\
\text { No llega difusión } \\
\text { ni comunicación a } \\
\text { todo el personal. } \\
\text { Qué acciones } \\
\text { realizar para } \\
\text { concienciar más } \\
\text { gente. }\end{array}$ & $\begin{array}{l}\text { Acciones } \\
\text { anteriores al Plan: } \\
\text { Humanización } \\
\text { en Pediatría y } \\
\text { Plan Estratégico } \\
\text { 2015-2019 con } \\
\text { eje destacado en } \\
\text { humanización. }\end{array}$ & $\begin{array}{l}\text { Presencia casi nula } \\
\text { de humanización } \\
\text { en la página. }\end{array}$ \\
\hline Infanta Cristina & $\begin{array}{l}\text { Muy directa y clara. } \\
\text { Intranet, agenda y } \\
\text { novedades. } \\
\text { A través de } \\
\text { Formación. } \\
\text { Trabajo coordinado } \\
\text { con Formación y } \\
\text { Comunicación. }\end{array}$ & $\begin{array}{l}\text { Integrantes comisión } \\
\text { impulsan acciones } \\
\text { en sus áreas. } \\
\text { Mensajes } \\
\text { humanización } \\
\text { en cartelería y } \\
\text { pantallas. }\end{array}$ & $\begin{array}{l}\text { Quejas por } \\
\text { demasiadas } \\
\text { actividades. } \\
\text { No todo personal } \\
\text { sanitario aplica } \\
\text { atención centrada en } \\
\text { el paciente. }\end{array}$ & $\begin{array}{l}\text { Campaña "Hospital } \\
\text { con Empatía". } \\
\text { Avances en } \\
\text { Experiencia del } \\
\text { paciente. }\end{array}$ & $\begin{array}{l}\text { Mucha presencia } \\
\text { humanización en la } \\
\text { página. }\end{array}$ \\
\hline
\end{tabular}

Fuente: Elaboración propia en base a las entrevistas mantenidas con responsables y coordinadores de acciones de humanización de los hospitales de la muestra 


\subsection{Los grupos de discusión}

6.3.1. Grupo de discusión realizado con el personal de enfermería de los siguientes servicios: Unidad de Cuidados Críticos, Quirófano, Atención al Paciente, Hospital de día y Pediatría

Nos llama la atención el diálogo sostenido por dos de los participantes:

- Participante A: “¿Y por qué llega ahora lo de la humanización?”

- Participante B: "Quizás porque nos estamos deshumanizando".

El concepto de humanización de la atención sanitaria está presente en el grupo.

Algunos participantes afirman realizar sus tareas diarias de forma humanizada. Entienden y expresan que la forma adecuada de emprender su trabajo es centrando la atención en el paciente y en los cuidadores (sobre todo de familiares). Pero también se desprende de sus comentarios que no es algo generalizado en todo el hospital. Y se refieren en varias ocasiones a la forma en que otros compañeros realizan su trabajo de forma "deshumanizada".

Consideran, sin embargo, que cuentan con formación suficiente sobre el tema, y que la comunicación sobre la formación relacionada con la humanización y la atención centrada en el paciente es frecuente y abundante, pero a veces "no llega al trabajador".

En general, se refieren a peculiaridades relacionadas con su trabajo como la cantidad y los riesgos de los trabajadores sanitarios, pues pueden incluso llegar al estrés, al burn out (síndrome de "estar quemado"), señalando la falta de herramientas para sobrellevar situaciones personales de tensión.

Y comentan espontáneamente la diferencia del comportamiento que se les exige a los distintos profesionales sanitarios. Del médico se espera un buen desarrollo científico técnico, y de la enfermera, el trato.

\subsubsection{Grupo de discusión realizado con médicos de un gran hospital.}

Uno de los participantes en el grupo de discusión inició su exposición con un sorprendente comentario:

A los profesionales les mandamos un mensaje perverso, les decimos por un lado que hay un Plan de Humanización, que hay que tratar bien a los pacientes, pero sólo se les pide que respondan por actividad. Al final tu plus de productividad depende de que hayas operado a tantos pacientes, hayas hecho tantas consultas, hayas... actividad, actividad, actividad. ¿Qué le interesa al jefe de servicio?, actividad, actividad, actividad. No le interesa que le hayas preguntado al paciente cómo está.

El grupo en general es crítico sobre los alcances de la humanización del sistema sanitario, y es crítico sobre el Plan de Humanización, especialmente por la gran cantidad de tareas administrativas que restan tiempo a la ejecución de otras propias.

Consideran que las acciones de humanización en el hospital, obtienen muy buenos resultados, pero se trata de propuestas previas o independientes al Plan. 
El grupo médico es muy crítico con la comunicación del Plan de Humanización en el hospital, la consideran insuficiente y de perfectible eficacia. En general, afirman que la comunicación interna es escasa: "Los mandos medios no transmiten, pero tampoco la información llega clara a esos niveles".

Los médicos se muestran conocedores e interesados por la humanización, pero no la consideran un estándar básico, "como por ejemplo la higiene de manos".

Cuestionan las encuestas de satisfacción, que siempre obtienen resultados por encima del 80 por ciento. Unos resultados percibidos como "un fracaso si uno no llega al 90 por ciento".

Conviene destacar la percepción de estos profesionales de la Medicina acerca del tipo de paciente. Un paciente informado, que empieza a preguntar y a reclamar, y quien va a forzar la mejora paulatina del sistema.

La participante más joven del grupo muestra una visión optimista. Se refiere a una nueva forma de ejercer la Medicina, ya humanizada. "Con estos nuevos profesionales, y sensibilizando a los más antiguos, el sistema sanitario tenderá a mejorar".

\section{Conclusiones}

Desde el Plan de Humanización del Insalud, de 1984, hasta el de la Comunidad de Madrid de 2016, los hospitales españoles instituyeron y difundieron en mayor o menor medida acciones de atención centrada en el paciente, que además del cuidado y la empatía, trajeron la escucha activa, la información y el empoderamiento del paciente en la toma de decisiones sobre su salud y su vida.

Sin embargo, y a pesar de la relevancia que el Plan de Humanización asigna a la comunicación, observamos, en los resultados de nuestra investigación, la inexistencia de una línea clara de actividades de comunicación de la humanización. Y, si bien se emprenden muchas actividades, como hemos podido comprobar, no se han establecido canales y propuestas específicas para llegar a todos los que forman parte de la plantilla de un hospital. Además, aunque el Plan reconoce la importancia de la cultura organizacional, el problema parece radicar en la falta de planificación y especificación de los canales para comunicar la humanización en los hospitales analizados.

Así, en los hospitales grandes el personal sanitario reclama mayor eficiencia comunicativa. Los mensajes no les llegan, "la gente no se entera”. Sólo se informa quien ya tenía inquietudes sobre este tema. Comprobamos, así, como la comunicación en el sector hospitalario no ha avanzado todo lo necesario.

La percepción de los representantes de los hospitales más grandes corrobora la conclusión de estudios anteriores. La magnitud de ciertos centros hospitalarios dificulta la comunicación entre estamentos del mismo nivel y constituye un inconveniente para el buen desarrollo de la comunicación vertical, por lo que muchos esfuerzos de comunicación interna se pierden por el camino.

Sin embargo, la comunicación de la humanización en los hospitales de menor tamaño sí se percibe eficaz: "sí se llega". Sin duda, contribuye a ello su estructura más reducida y menos compleja y una comunicación mucho más directa. 
Una futura línea de trabajo, derivada de este estudio, podría plantearse en el ámbito de los hospitales grandes, con los mandos intermedios y los líderes informales de los distintos servicios, con el objetivo de convertirlos en verdaderos embajadores de la humanización en el propio hospital. Potenciar y capacitar a este personal en la forma de transmitir la información en un doble sentido, tanto a los subordinados, como a los superiores, podría redundar en una mejora en la calidad de la comunicación de los grandes hospitales.

Como hemos comprobado, la comunicación hospitalaria se reviste de ciertas complejidades particulares. En primer lugar, los hospitales son organizaciones en las cuales existen muchas formas de entrar en comunicación con los usuarios. Y, en segundo lugar, los trabajadores están agrupados en estructuras formadas por distintos tipos de profesionales. Tras escuchar a los médicos y al personal de enfermería en el transcurso de los grupos de discusión, se observa un alto nivel de corporativismo entre los diferentes profesionales de los hospitales. El "nosotros" y el "ellos", siendo los otros los médicos, enfermeros, auxiliares o celadores, se hace presente con mucha fuerza en las expresiones de los profesionales sanitarios.

Pese al afán por innovar de las personas que coordinan las actividades en los hospitales grandes, medianos y pequeños, en su deseo de encontrar nuevas formas de acercar la humanización a cada trabajador del hospital, los datos del Observatorio del Servicio Madrileño de Salud, sobre satisfacción de la atención al paciente en 2018, resultan ser, en general, algo inferiores a los de 2017.

Podría pensarse, como una de las causas de esta reducción, que el sistema sanitario está sobrecargado, con más pacientes, más consultas, los médicos no dan abasto, tienen menos tiempo y ello podría llevarles a prestar una atención escasa al paciente.

O quizás, la respuesta esté en los propios pacientes, quienes empiezan a dar por sentados ciertos estándares y a exigir cada día más en la prestación de los servicios hospitalarios, con la presión que ello supone para los facultativos y todo el personal sanitario.

Descubrimos así, otra futura línea de investigación, la de trabajar con las asociaciones de pacientes y con los ciudadanos en general, para conocer su percepción sobre la humanización, y desarrollar campañas para potenciar la figura del "paciente informado". Informado, sí, pero respetuoso, consciente y empático con las circunstancias particulares determinantes del ejercicio de las profesiones sanitarias.

Y una tercera línea de investigación pertinente sería un trabajo comparativo entre los servicios sanitarios de las distintas Comunidades Autónomas de España y sus avances en cuanto a humanización. La Red Sanitaria de Responsabilidad Social, con su creciente expansión nacional, podría ser un interlocutor válido para este trabajo.

Concluimos que la atención centrada en el paciente, la atención humanizada, es la forma idónea de brindar los servicios del sistema de salud. En algún momento nos costará recordar que hubo otra forma de prestar el servicio hospitalario. Para lograrlo, y dotar al sistema sanitario de las necesarias herramientas y estrategias comunicativas, resulta imprescindible la investigación desde la comunicación y las relaciones públicas. 


\section{Referencias bibliográficas}

Balint, M. (1979). El médico, el paciente y la enfermedad. Buenos Aires: Paidos.

Bermejo, J. C. (2014). Humanizar la asistencia sanitaria: Aproximación al concepto. Bilbao: Desclée de Brouwer.

Bertalanffy, L. v. (1976). Teoría general de los sistemas. México: Fondo de Cultura Económica.

Bustos, F. (2017). El bien intangible Responsabilidad Social Institucional. En Canel, M. J., Piqueiras, P. y Ortega, G. (Eds.) La comunicación de la Administración Pública: conceptos y casos prácticos de bienes intangibles (pp. 123-164). Madrid: INAP.

Canyelles, J. M. (2011). Responsabilidad social de las administraciones públicas. Revista de Contabilidad y Dirección, 13(7), 77-104

Centro de Investigaciones Sociológicas (2018). Barómetro Sanitario. Disponible en: http://bit.ly/2UmKm3p [Consultado el 19/6/2019].

Costa Sánchez, C. (2012a). El gabinete de comunicación del hospital. Propuesta teórica y acercamiento a la realidad de los departamentos de comunicación de los hospitales públicos de Galicia. Doxa Comunicación, 14, 175-197. DOI: 10.31921/ doxacom

Costa Sánchez, C. (2012b). Estrategias de comunicación corporativa. En Medina, P. y Pacanowski, T. (Eds.), Comunicación hospitalaria: un plan para el siglo XXI (pp. 107-125). Madrid: Fragua.

Dirección General de Coordinación de la Atención al Ciudadano y Humanización de la Asistencia. (2017). Marco estratégico de promoción de la Responsabilidad Social Sociosanitaria. Madrid Salud. Consejería de Sanidad de la Comunidad de Madrid. Disponible en: http://bit.ly/2jZWf1z [Consultado el 20-07-2019].

E-Grunig, J.; A-Grunig, L.; Aparecida-Ferrari, M. (2015). Perspectivas de las Relaciones Públicas: resultados del Excellence Study para la comunicación en las organizaciones. Revista Mediterránea de Comunicación 6(2). 9-28. DOI: 10.14198/ MEDCOM2015.6.2.01

En Sanidad, mejor Responsabilidad Social Sociosanitaria que Corporativa. (17 de marzo de 2014). Redacción Médica. https://www.redaccionmedica.com/noticia/en-sanidad-rsc-o-responsabilidad-social-sociosanitaria-1763

García Nieto, M. T. (2012a). Las Ciencias Sociales y la Responsabilidad Social Corporativa. aDResearch ESIC: International Journal of Communication Research, 6(6) 92-111.

García Nieto, M. T. (2012b). La comunicación con los públicos internos. En Medina, P. y Pacanowski, T. (Eds.), Comunicación hospitalaria: un plan para el siglo XXI (pp. 127-150). Madrid: Fragua.

Gil Paz, I.; González Martínez, P.; López Acón, A.; Montes Vázquez, S.; Torres Insua, R.; Caamaño Ponte, J.; Fernández Nistal; J. (2018). La humanización de la asistencia sanitaria y su comunicación a través de la responsabilidad social. Revista Española de Comunicación en Salud. VOL. 9 (1), 54-63. DOI: https://doi.org/10.20318/recs.2018.4254

Greene, S.M., Tuzzio, L., y Cherkin, D. (2012). A Framework for Making PatientCentered Care Front and Center. The Permanent Journal, 16(3), 49-53. 
Grunig, J. (1992). Communication, Public Relations, and Effective Organizations: An Overview of the Book. En Grunig, J. (Ed.), Excellence in Public Relations and Communication Management (pp. 1-28). New Jersey: Lawrence Erlbaum Associates.

Grunig, J. E. y Grunig, L. A. (2008). Excellence Theory in Public Relations: Past, Present, and Future. In Public Relations Research (pp. 327-347). Wiesbaden: VS Verlag für Sozialwissenschaften. DOI: 10.1007/978-3-531-90918-9-22

Grunig, J., Grunig, L. y Ehling, W. (1992). What is an Effective Organization? En Grunig, J. (Ed.), Excellence in Public Relations and Communication Management (pp. 65-90). New Jersey: Lawrence Erlbaum Associates

Grunig, J. y Hunt, T. (2003). Dirección de relaciones públicas. Barcelona: Gestión 2000.

INIDRESS (2017). Decálogo de Responsabilidad Social Sanitaria y Sociosanitaria. Recuperado el de: http://bit.ly/30LaZS9 [Consultado el 07-06-2019].

King, A. y Hoppe, R. B. (2013). "Best practice” for Patient-Centered Communication: A Narrative Review. Journal of Graduate Medical Education, 5(3), 385-393.

Lancet (2018). Measuring performance on the Healthcare Access and Quality Index for 195 countries and territories and selected subnational locations: a systematic analysis from the Global Burden of Disease Study 2016. Lancet No391, 22362271. May 23, 2018. DOI: https://doi.org/10.1016/S0140-6736(18)30994-2

Medina, P. y Pacanowski, T. (Eds.) (2012). Comunicación hospitalaria: un plan para el siglo XXI. Madrid: Fragua.

Ministerio de Sanidad y Consumo (1984). Plan de Humanización de la Asistencia Hospitalaria. Disponible en: http://bit. ly/32jj94t [Consultado el 19-08-2019].

Preston, L. E. y Post, J. E. (1975): Private Management and Public Policy: The Principle of Public Responsibility. Englewood Cliffs, N.J: Prentice-Hall, pp. 24-27.

Quintana Pujalte, A. L.; Castillo Esparcia, A.; Carretón Ballester, M. C. (2018). Relaciones Públicas ciudadanas. Actores, discursos y construcción de identidad de movimientos contra los desahucios en España. Obra digital: revista de comunicación $\mathrm{N}^{\mathrm{o}}$. 15, 83-97. DOI: 10.25029/od.2018.197.15

Red Sanitaria de RSC y Grupo Innova-Docencia no 176 (2018). Una innovadora propuesta de responsabilidad social en la sanidad pública española. En Gaona Pisonero, C. (Coor.), Temáticas emergentes en innovación universitaria (173-187). Madrid: Tecnos.

Rodríguez Cala, A. (2017): Responsabilidad Social Corporativa en la Red Hospitalaria de Utilización Pública de Cataluña. Tesis doctoral. Universitat de Lleida. http://hdl.handle.net/10803/418804

Salcedo de Prado, M. (2012). La comunicación en el contexto hospitalario. En Medina, P. y Pacanowski, T. (Eds.), Comunicación hospitalaria: un plan para el siglo XXI (pp. 36-47). Madrid: Fragua

Stewart, M. (1995). Effective Physician-Patient Communication and Health Outcomes: A Review. CMAJ: Canadian Medical Association Journal, 152(9), 1423-1433.

Stewart, M. (2001). Towards a Global Definition of Patient Centred Care. BMJ. Clinical research ed, 322(7284), 444-445. 
Stewart, M.; Brown, J. B.; Weston, WW.; McWhinney, I. R.; McWilliam, C. L. y Freeman, T. R. (2003). Patient-Centered Medicine. Transforming the clinical method. Abingdon: Radcliffe Medical Press Lted. Second ed.

Subdirección General de Humanización de la Asistencia Sanitaria (2016) Plan de Humanización de la asistencia sanitaria 2016-2019. Disponible en: http://bit.ly/2ZBGroC [Consultado el 01-06-2019].

Viñarás Abad, M.; Cabezuelo Lorenzo, F. (2012). Los stakeholders de la RSC desde la perspectiva de las relaciones públicas: estudio del caso de tres compañías internacionales. Hologramatica N. 16 (2), 37-61. Disponible en: www.hologramatica. com.ar

Zarco, J. (2018a). Julio Zarco, presidente de la Fundación Humans: "La humanización ha venido para transformar el SNS”. Portal ISanidad. , 7 de septiembre. Disponible en: 1908-19 de: http://bit.ly/2LjMyok [Consultado el 30-05-2019].

Zarco, J. (2018b). Humanización de la sanidad: perspectiva médica. En Sánchez-Caro, J. y Abellán, F. (Coords.), Avances en salud: aspectos científicos, clínicos, bioéticos y legales (pp. 207-212). Madrid: Fundación Merck.

Zarco, J. (2019). Hacia dónde va la humanización de la atención sanitaria. Jornada Humanización en la atención sanitaria en el Hospital Universitario Gregorio Marañón, Madrid, 26 de marzo. 